\title{
A systematic review of parent/peer-based group interventions for adolescents with type 1 diabetes: interventions based on theoretical/therapeutic frameworks
}

\author{
KALSOOM AKHTER, ${ }^{1}$ TRIECE TURNBULL, ${ }^{2}$ DAVID SIMMONS ${ }^{3}$
}

\begin{abstract}
Objective: Although interventions which provide psychosocial support can have a positive impact on diabetes self-care, the impact of family/peer- and theory-based interventions has not yet been clearly identified. This systematic review investigates the randomised controlled trials (RCTs) employing family/peer-based interventions (based on theoretical/ therapeutic frameworks) which aim to improve adolescents' glycaemic control, psychosocial and/or behavioural functioning.

Methods: The Cochrane Library, database of systematic reviews, database of abstracts of reviews of effectiveness and Health Technology Assessment database were searched (from start date until February 2016) for any previously conducted systematic reviews. Seventeen RCTs/interventions were included. The literature was also identified by contacting the leading researchers. Glycaemic control was measured by $\mathrm{HbA}_{1 \mathrm{c}}$ and psychosocial functioning by measures of selfcare, knowledge and communication, collaboration/teamwork, quality of life, problem solving, social functioning and family functioning. Only those interventions which reported the use of theories/therapies to manage type 1 diabetes and other psychosocial issues among adolescents (aged 12-17) were included in the present review. Data summarising the key features of the interventions was extracted from each article. Where possible, the effect sizes were calculated.

Results: The effect sizes could be computed for $\mathrm{HbA}_{1 \mathrm{c}}$ in six of the 17 interventions. The overall outcomes indicated that interventions including parents have a small to large effect size on a variety of diabetes management and psychosocial
\end{abstract}

Department of Psychology, City University London, London, UK

Faculty of Health and Society, University of Northampton, Northampton, UK

School of Medicine, Western Sydney University, Campbell town, New

South Wales, Australia

Address for correspondence: Dr Kalsoom Akhter

School of Arts and Social Sciences, Department of Psychology, City

University London, Northampton Square, London EC1V OHB, UK

E-mail: kalsoom.akhter.1@city.ac.uk

https://doi.org/10.15277/bjd.2018.177 outcomes. This review identified interventions, mostly including parents and rarely including peers.

Conclusion: The results of this systematic review demonstrate that multicomponent interventions may be more successful for adolescents than ones that just focus on one aspect. Effectiveness is also greater if they demonstrate inter-relatedness with the various aspects of diabetes management. Short-term behavioural approach-based interventions promote improvements in parent/adolescent relationships. Outreach home-based interventions could be a more accessible alternative for intervening with families than office/hospital-based interventions. This approach may also be more acceptable to adolescents and their families. There is a need to develop evaluated interventions for adolescents involving parents. Development should involve stakeholders (ie, adolescents, their families and healthcare professionals) to co-design potentially cost-effective and feasible interventions in the context of NHS diabetes services. Br J Diabetes 2018;18:51-65

Key words: family/peer-based interventions, psychosocial and behaviouraltheories/therapies, diabetes and psychosocial functioning, type 1 diabetes, adolescents

\section{Introduction}

Type 1 diabetes requires managing an intensive and challenging regimen. This includes integration of daily medical tasks (insulin injections, frequent blood glucose tests) and lifestyle modifications (close monitoring of food intake and regular exercise) in everyday life. 1,2 Although near-normal blood glucose control may decrease the risk of several long-term diabetes complications in individuals with type 1 diabetes, ${ }^{3,4}$ adolescents often have difficulty juggling all aspects of such a demanding treatment regimen. Parents and other family members are especially important for adolescents with type 1 diabetes 5 to encourage and provide support for treatment-related behaviours. ${ }^{2}$ Anderson and colleagues ${ }^{6}$ showed that a low-intensity office-based family intervention for youths with type 1 diabetes increased parental involvement, while decreasing diabetes-related family conflict. 6,7

Research has highlighted that, during adolescence, young adults strive for autonomy and parents' attempts to monitor/control 
their children's treatment may be viewed as intrusive or nagging. Such attempts may result in adolescents becoming resistant, defiant and non-compliant. 8,9 Research supports the need for adolescents and parents to work cooperatively, with open communication and flexible problem-solving skills, in order to negotiate shared responsibility for treatment management. ${ }^{10-12}$

On the other hand, research has shown that age needs to be considered and that support needs to occur at both home and school. Consequently, both family members and peers can facilitate optimal diabetes management. ${ }^{2}$ Support from peers has been rated as important by adolescents with type 1 diabetes ${ }^{13}$; peers are more likely than family members to provide companionship and emotional support in relation to diabetes care. ${ }^{14}$ In one structured group intervention, adolescents and their friends demonstrated higher levels of knowledge about diabetes and support, as well as a higher ratio of peers to family support. In addition, parents reported a decrease in diabetes-related conflict. ${ }^{15}$ Nonetheless, the shift from parental to peer support is not absolute, as adolescents continue to seek guidance and advice from their parents. ${ }^{16}$ Numerous theoretical and therapeutic frameworks have been devised to explain and adapt the relationship between patients' perceptions and their behaviour (see Box 1 for a brief overview). ${ }^{17}$

\section{Box 1 Theoretical frameworks included in the review}

\section{Self-regulation model}

The Self-Regulation Model of Illness (SRMI) provides a framework for understanding how individual symptoms and emotions experienced during a health threat or diagnosis influence perception of illness and guide subsequent coping behaviour. ${ }^{18,19}$ The SRMI may be useful to further understanding of type 1 diabetes patients' behaviours related to their condition and psychosocial functioning. The SRMI theory suggests that individuals search to understand their illness or disease threat by developing an understanding of what the illness is, what it means, its causes, its consequences, how long it will last, and whether it can be cured or controlled. This understanding (or illness representation) is not necessarily scientifically or medically validated, but formulated from personal experience (physical symptoms and emotions), social influences, and/or interaction with healthcare providers. Individuals are thought to reduce their health risk or change their health behaviour in ways consistent with their own illness representation. ${ }^{20}$

\section{Empowerment approach}

The goal of patient empowerment is to build up the capacity of patients to help them to become active partners in their own care, to enable them to share in clinical decision making, and to contribute to a wider perspective in the healthcare system. ${ }^{21}$ Empowerment is a positive concept that refers to the patient's facilities, abilities and surrounding environment. ${ }^{21}$ Power is an inner feeling of self-awareness and self-education. ${ }^{22}$ Empowerment is achieved through interaction between people and causes interpersonal and intrapersonal communications. ${ }^{23}$ It is a practical strategy in improving health conditions, ${ }^{24}$ which includes skills (eg, solving problems, boosting self-confidence and creating strategies to create mutual trust). ${ }^{25}$ The empowerment process begins with providing the patient with information and education and ends when he/she can actively participate in making 'smart' decisions about his/her disease. ${ }^{26}$ With this approach, health professionals facilitate patients to make informed decisions regarding their particular conditions. Patients are encouraged to fully participate in their treatment process by sharing their knowledge and experiences and making decisions through mutual assistance. Empowerment discovers and expands one's inner capacity to accept responsibility towards their health. It is an intervention or a strategy to help people change their behaviour in order to adhere to the treatment plan. ${ }^{27}$

\section{Self-efficacy theory}

Self-efficacy (SE) was first proposed by Albert Bandura, who suggested that self-efficacy is a belief of individuals in their abilities to carry out a successful practice and is a theory in itself, as well as a structure of the social cognitive theory. The self-efficacy theory argues that people will take action when they believe they are able to do it and will avoid actions when they believe they may fail. Self-efficacy is the prerequisite of a behaviour and should be considered as an independent part of basic skills. ${ }^{28}$ In total, Bandura believes that self-efficacy (or 'mastery') is the main structure in predicting individuals' behaviour change and usually the ones that show a high level of behavioural changes have higher efficacy. ${ }^{29}$ Self-efficacy has a prominent role in diabetes self-management and predicts its outcome. ${ }^{30}$

\section{Social Cognitive Theory, Social Learning Theory}

Social Cognitive Theory (SCT) started as the Social Learning Theory (SLT) in the 1960s by Albert Bandura and it was developed into the SCT in 1986 SCT is based on the concept that learning is affected by cognitive, behavioural and environmental factors. ${ }^{31}$ In contrast to the traditional psychological theories that emphasised learning through direct experience, Bandura posited that virtually all learning phenomena can occur by observing other people's behaviour and its consequence. ${ }^{32}$

\section{Positive psychology}

Positive psychology suggests understanding the role of positive traits, experiences and environmental factors that contribute to wellness. Given the wide variability in how patients with chronic illness are able to manage their daily behaviours needed to maintain physical and mental well-being, positive psychology has provided a particularly useful framework to identify the factors that promote successful disease management. ${ }^{33}$

\section{Theoretical frameworks underlying some therapeutic approaches}

Social ecology theory underlying Multi-Systemic Therapy (MST)

Bronfenbrenner's theory of social ecology ${ }^{34}$ serves as the conceptual foundation of MST. Youth are viewed as nested within multiple systems (eg, family, peer, school, neighbourhood) that have direct (eg, parenting practices) and indirect (eg, neighbourhood context affects parenting practices) influences on behaviour and interactions among individuals are reciprocal in nature. On a clinical level, several relatively compatible theoretical perspectives have influenced the development of interventions used in MST. These include Minuchin's structural formulations (eg, attention to boundaries and repeated patterns of interaction that regulate family members' behaviours), ${ }^{35}$ Haley's strategic formulations (eg, importance of understanding recursive sequences of behaviour and family hierarchy), ${ }^{36}$ social learning theory (eg, importance of modelling and reinforcement in influencing behaviour) and cognitive behavioural theory (eg, problem solving skills). Interventions derived from these approaches are integrated into the broader social ecological framework that underlies MST. ${ }^{37}$

Behavioural and system models underlying Behavioural Family System herapy (BFST)

Two predominant models of family functioning are the behavioural and systems models. Behaviourally oriented theorists have explained family processes in terms of molecular contingency arrangements, social learning principles and behaviour exchange theory. ${ }^{38-40}$ Family systems theorists have explained these same processes in terms of circular cybernetic systems with an emphasis on molar-level structural analysis. ${ }^{41,42}$ While there are definite differences between these two theoretical approaches, they both share a common emphasis on observable regularities in interpersonal processes. Robin and Foster ${ }^{43}$ have integrated concepts from both schools in building a comprehensive behavioural systems theory of family functioning, adding notions of contingency arrangements, social learning principles and cognitive behavioural theory to the analysis of the family as a circular system with a definite structural configuration. 
No systematic review exists relating to the currently explored area. Therefore, the purpose of this systematic review is to investigate the effects of family/peer-based interventions (involving theoretical frameworks) on type 1 diabetes management and psychosocial functioning among adolescents.

\section{Methods}

\section{Eligibility criteria}

Studies were included if they were RCTs, used any theoretical/therapeutic frameworks and included adolescents with type 1 diabetes (age $<18$ years), their peers or friends and parents or families. Two domains of outcome measures were extracted: diabetes functioning/medically performing/HbA1c and psychosocial functioning. Studies were excluded if they involved improving education alone, had no explicit theoretical background, 6,7,44-46 were not a RCT, 15,4650 included children only ${ }^{51,52}$ or adults ${ }^{53}$ and did not involve parents or peers.

\section{Search strategy and data extraction}

A systematic computerised search was performed in CINAHL, E-journals, Econlit library, Information Science and Technology abstracts, Psycharticles, Socindex, Medline, PsychInfo, Embase, Psychological and Behavioural Sciences Collection, Socindex and Cochrane Central Register of Controlled Trials (from their start date to February 2016). The National Research Register database was searched for unpublished literature and information on unpublished or in-progress research was requested via contacting leading authors. The reference lists of the retrieved studies and other key reviews (eg, Hampson et al, ${ }^{54}$ ) were checked in order to capture other relevant publications (see Appendix A for search terms at www.bjd-abcd.com).

The first researcher independently reviewed all titles and abstracts in two phases. First, the retrieved titles and abstracts were reviewed to identify relevant studies. The full texts of retrieved studies were then read to determine eligibility. A second researcher reviewed all the articles in order to determine the inter-rater reliability in inclusion/exclusion of studies. Any discrepancies or differences in opinion were resolved by consensus. Information from the included articles was extracted using a standardised form. The extraction process identified the following areas from each article: study and country, study population, theoretical frame work, time to follow-up, outcome measures and results.

\section{Quality assessment}

A tool to assess the methodological quality of quantitative studies was used, which was developed and tested by the Effective Public Health Practice Project. ${ }^{55}$ Each study was appraised according to the six criteria and rated as 'strong', 'moderate' or 'weak'. The reviewers independently scored all relevant articles for methodological quality. Any discrepancies were resolved through discussion. Ten studies were strong and seven were moderate, ${ }^{58,59,63-65,70,71}$ with none being weak in terms of their quality (see Table 1).

\section{Results}

\section{Search results}

The search of the databases retrieved 3,074 records. Following broad and narrow screening, 17 papers were considered suitable for inclusion in the review. ${ }^{56-72}$ A study flow diagram of included and excluded studies is provided in Figure 1.

Included studies also differed with regard to the theories, durations and outcome measures (see Table 2). Due to this heterogeneity, it was not possible to perform a meta-analysis. Instead, findings are presented in a narrative format.

\section{Study characteristics}

The selected studies varied considerably with regard to their study

Table 1 Quality assessment results for methodologically relevant studies $(n=17)$

\begin{tabular}{|c|c|c|c|c|c|c|c|}
\hline $\begin{array}{l}\text { Study } \\
\text { reference }\end{array}$ & $\begin{array}{l}\text { Selection } \\
\text { bias }\end{array}$ & $\begin{array}{l}\text { Study } \\
\text { design }\end{array}$ & Confounders & Blinding & $\begin{array}{l}\text { Data collection } \\
\text { methods }\end{array}$ & $\begin{array}{l}\text { Withdrawals/ } \\
\text { dropouts }\end{array}$ & $\begin{array}{l}\text { Global } \\
\text { rating }\end{array}$ \\
\hline 56 & Strong & Strong & Moderate & Strong & Strong & Moderate & Strong \\
\hline 57 & Moderate & Strong & Moderate & Moderate & Strong & Strong & Strong \\
\hline 58 & Strong & Strong & Moderate & Weak & Strong & Strong & Moderate \\
\hline 59 & Moderate & Strong & Weak & Moderate & Strong & Strong & Moderate \\
\hline 60 & Strong & Strong & Strong & Strong & Strong & Strong & Strong \\
\hline 61 & Moderate & Strong & Strong & Strong & Strong & Strong & Strong \\
\hline 62 & Strong & Strong & Moderate & Moderate & Strong & Strong & Strong \\
\hline 63 & Moderate & Strong & Moderate & Moderate & Strong & Strong & Moderate \\
\hline 64 & Strong & Strong & Weak & Moderate & Strong & Moderate & Moderate \\
\hline 65 & Strong & Strong & Strong & Moderate & Strong & Weak & Moderate \\
\hline 66 & Strong & Strong & Moderate & Moderate & Strong & Strong & Strong \\
\hline 67 & Strong & Strong & Moderate & Moderate & Strong & Strong & Strong \\
\hline 68 & Strong & Strong & Moderate & Moderate & Strong & Strong & Strong \\
\hline 69 & Strong & Strong & Moderate & Moderate & Strong & Strong & Strong \\
\hline 70 & Strong & Strong & Weak & Moderate & Strong & Strong & Moderate \\
\hline 71 & Strong & Strong & Weak & Moderate & Strong & Strong & Moderate \\
\hline 72 & Moderate & Strong & Moderate & Moderate & Strong & Moderate & Strong \\
\hline
\end{tabular}


Figure 1. Flow diagram of studies.

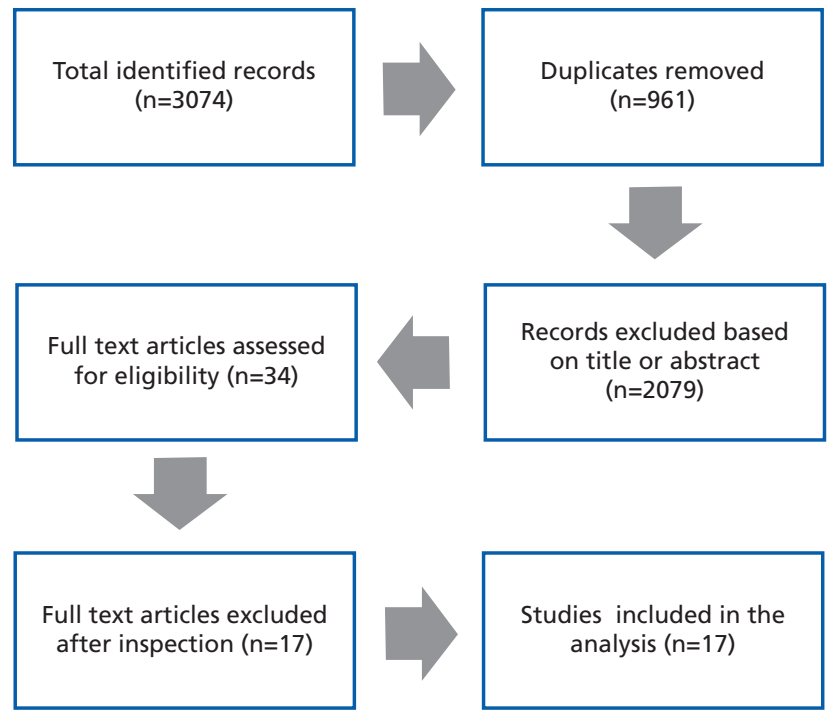

characteristics (see Appendix B at www.bjd-abcd.com). Studies were conducted between 1985 and 2014, but the majority were published after 2000.

\section{Efficacy of theory-based interventions on diabetes and psychosocial functioning}

The majority of the reviewed studies involved parents/families except two involving peers. ${ }^{62,70}$ The reviewed studies were groupbased except the one by Newton and Ashley, ${ }^{70}$ which was a webbased intervention. This section will draw attention towards the efficacy of the interventions based on various theories/therapies mentioned in Box 1. These theories/therapies were used to understand the behaviours influencing the diabetes and psychosocial functioning of the trial participants.

\section{Social learning and self-efficacy theory (involving peers and} adolescents)

Two studies used and evaluated the value of both theories. ${ }^{62,70}$ The study by Kaplan et a/62 was based on social learning and selfefficacy theory 29 to improve social learning skills and resist peer pressure. Training based on theoretical frameworks produced better glycaemic control at 4 months compared with the control group. There were positive (but non-significant) correlations between social support satisfaction and $\mathrm{HbA}_{1 c}$, as well as knowledge and $\mathrm{HbA}_{1 \mathrm{c}}$. Similarly, there was a positive and significant correlation between the Mean Ends Problem Solving test (MEPS) and $\mathrm{HbA}_{1 c}(p<0.01)$. These correlations suggest that those who were most satisfied with their networks of social support actually had the poorest glycaemic control. The results of this study also highlight the importance of behaviour in the management of type 1 diabetes rather than just focusing upon knowledge.

Similarly, Newton and Ashley ${ }^{70}$ conducted a web-based intervention using self-efficacy theory to improve compliance with treat- ment protocols and psychosocial functioning. This website seemed to provide problem-solving activities, knowledge about diabetes and a source of social support as well. Self-efficacy was significantly correlated with positive outcome expectations, diabetes self-management and quality of life for youths. In an exit survey, $90 \%$ of participants indicated that they were more willing to comply with their treatment protocol after participating in the intervention.

Empowerment approach (involving parents and adolescents) The study by Viklund and colleagues ${ }^{63}$ aimed to determine the effects of an empowerment programme on glycaemic control and empowerment and to study the role of parental involvement. Overall, this empowerment programme showed no beneficial glycaemic or empowerment effects 6 and 12 months after the intervention.

Social cognitive theory, self-regulation model (families and adolescents)

Nansel and colleagues $^{61}$ designed a multicentre group study grounded in social cognitive and self-regulation theories to help families improve diabetes management by facilitating problem-solving skills, communication skills and appropriate responsibility sharing. A significant improvement in glycaemic control was seen in the 12-14-year age group from baseline at the 24-month interval, but not in adherence. ${ }^{61}$

Positive psychology (involving parents and adolescents) Jaser and colleagues ${ }^{71}$ focused on testing the feasibility and acceptability of a positive psychology intervention to improve adherence. No main effects for treatment were observed at the 6-month follow-up. However, there was a significant association between adolescents' levels of positive effect and measures of adherence, including self-report and meter downloads of glucose monitoring. Overall, the high levels of participation and retention indicate that adolescents and their parents were receptive to a positive psychology approach, ${ }^{10}$ which places an emphasis on positive emotions and strengths rather than problems. ${ }^{71}$

\section{Behavioural systems theory (of family functioning) underlying Behavioural Family Systems Therapy (families and adolescents)}

This theory is a combination of behavioural and family systems models (see Box 1) and its efficacy is demonstrated through Behavioural Family Systems Therapy (BFST). Seven studies ${ }^{59,64-69}$ evaluated the application of BFST for families of adolescents with diabetes. BFST is based on psychosocial principles of targeting family communication and problem-solving factors. ${ }^{43}$ Harris and colleagues ${ }^{59}$ examined whether home-based BFST produced clinically significant changes in family conflict in a sample of adolescents with poorly controlled diabetes and their families. The findings of this study suggested improvements in mothers' and adolescents' reported diabetes-specific conflict, as well as parentreported general parent/adolescent conflict.

Wysocki et a/64 compared the social validity of the BFST and Education Support (ES) group interventions as treatments for communication, problem-solving and conflict-resolution skills. 
Table 2 Data extraction

\begin{tabular}{|c|c|c|c|c|c|c|}
\hline $\begin{array}{l}\text { Study/ } \\
\text { country }\end{array}$ & $\begin{array}{l}\text { Study } \\
\text { population }\end{array}$ & $\begin{array}{l}\text { Theoretical } \\
\text { framework }\end{array}$ & $\begin{array}{l}\text { Time to } \\
\text { follow-up }\end{array}$ & $\begin{array}{l}\text { Outcome } \\
\text { measures }\end{array}$ & $\begin{array}{l}\text { Effect } \\
\text { sizes }\end{array}$ & Results \\
\hline 64/USA & $\begin{array}{l}\mathrm{N}=119 \\
2 \text { groups: } \\
\text { ES } \\
\text { BFST }\end{array}$ & BFST & $\begin{array}{l}3 \text { months } \\
6 \text { and } 12 \\
\text { months (results } \\
\text { not reported) }\end{array}$ & $\begin{array}{l}\text { Treatment evaluation } \\
\text { questionnaire }\end{array}$ & $x$ & $\begin{array}{l}\text { BFST was rated significantly more positively by } \\
\text { parents and/or adolescents. Adolescents rated ES } \\
\text { less positively than did parents }\end{array}$ \\
\hline \multirow[t]{17}{*}{$65 /$ USA } & $\begin{array}{l}N=119 \\
3 \text { groups: } \\
\text { BFST } \\
\text { ES } \\
\text { CT }\end{array}$ & BFST & $\begin{array}{l}3 \text { months } \\
6 \text { and } 12 \\
\text { months (results } \\
\text { not reported) }\end{array}$ & $\begin{array}{l}\text { PARQ: } \\
\text { Overt conflict/skill deficits } \\
\text { Extreme beliefs }\end{array}$ & $\begin{array}{l}-0.13 \\
-0.19 \\
-0.53 \\
-0.54\end{array}$ & \multirow[t]{17}{*}{$\begin{array}{l}\text { Compared with CT and ES, BFST yielded more } \\
\text { improvement in parent/adolescent relations and } \\
\text { reduced diabetes-specific conflict. Effects on } \\
\text { psychological adjustment to diabetes and diabetes } \\
\text { control were less robust. There were no effects on } \\
\text { treatment adherence }\end{array}$} \\
\hline & & & & $\begin{array}{l}\text { Family structure } \\
\text { Issues checklist: }\end{array}$ & $\begin{array}{l}-0.15 \\
-0.22\end{array}$ & \\
\hline & & & & $\begin{array}{l}\text { Number of items } \\
\text { endorsed }\end{array}$ & $\begin{array}{l}-0.18 \\
-0.20\end{array}$ & \\
\hline & & & & Total frequency of conflict & $\begin{array}{l}-0.27 \\
-0.30\end{array}$ & \\
\hline & & & & Total intensity of conflict & $\begin{array}{l}-0.13 \\
-0.31\end{array}$ & \\
\hline & & & & $\begin{array}{l}\text { Recall interview } \\
\text { conflict scores: } \\
\text { Frequency }\end{array}$ & $\begin{array}{l}0.07 \\
0.04\end{array}$ & \\
\hline & & & & Intensity & $\begin{array}{l}-0.21 \\
-0.03\end{array}$ & \\
\hline & & & & Duration & $\begin{array}{l}-0.21 \\
-0.13\end{array}$ & \\
\hline & & & & DRC & $\begin{array}{l}-0.09 \\
-0.19\end{array}$ & \\
\hline & & & & TADS & $\begin{array}{l}-0.35 \\
-0.30\end{array}$ & \\
\hline & & & & $\begin{array}{l}\text { Recall interview } \\
\text { adherence factors: } \\
\text { Insulin }\end{array}$ & $\begin{array}{l}0.20 \\
0.11\end{array}$ & \\
\hline & & & & $\begin{array}{l}\text { Testing/eating } \\
\text { frequency }\end{array}$ & $\begin{array}{l}0.34 \\
0.66\end{array}$ & \\
\hline & & & & $\begin{array}{l}\text { Diet } \\
\text { composition }\end{array}$ & $\begin{array}{l}0.48 \\
0.47\end{array}$ & \\
\hline & & & & $\begin{array}{l}\text { Diet } \\
\text { amount }\end{array}$ & $\begin{array}{l}-0.56 \\
-0.27\end{array}$ & \\
\hline & & & & Exercise & $\begin{array}{l}0.12 \\
0.01\end{array}$ & \\
\hline & & & & $\mathrm{SCl}$ & $\begin{array}{l}-0.28 \\
-0.24\end{array}$ & \\
\hline & & & & $\mathrm{HbA}_{1 \mathrm{c}}$ & $\begin{array}{l}0.19 \\
0.25\end{array}$ & \\
\hline 66/USA & $\begin{array}{l}\mathrm{N}=119 \\
3 \text { groups: } \\
\text { BFST } \\
\text { ES } \\
\text { CT }\end{array}$ & BFST & $\begin{array}{l}3 \text { months } \\
6 \text { months } \\
12 \text { months }\end{array}$ & $\begin{array}{l}\text { PARQ } \\
\text { TAD } \\
\text { DRC } \\
\text { SCl } \\
\mathrm{HbA}_{1 \mathrm{C}}\end{array}$ & $x$ & $\begin{array}{l}\text { Compared with CT and ES, BFST yielded lasting } \\
\text { improvements in parent/adolescent } \\
\text { relationships and diabetes-specific conflict. } \\
\text { Delayed effects on treatment adherence emerged } \\
\text { at } 6 \text { - and } 12 \text {-month follow-ups. There were no } \\
\text { immediate or delayed effects on adolescents' } \\
\text { adjustment to diabetes or diabetic control }\end{array}$ \\
\hline
\end{tabular}


Table 2 Data extraction continued

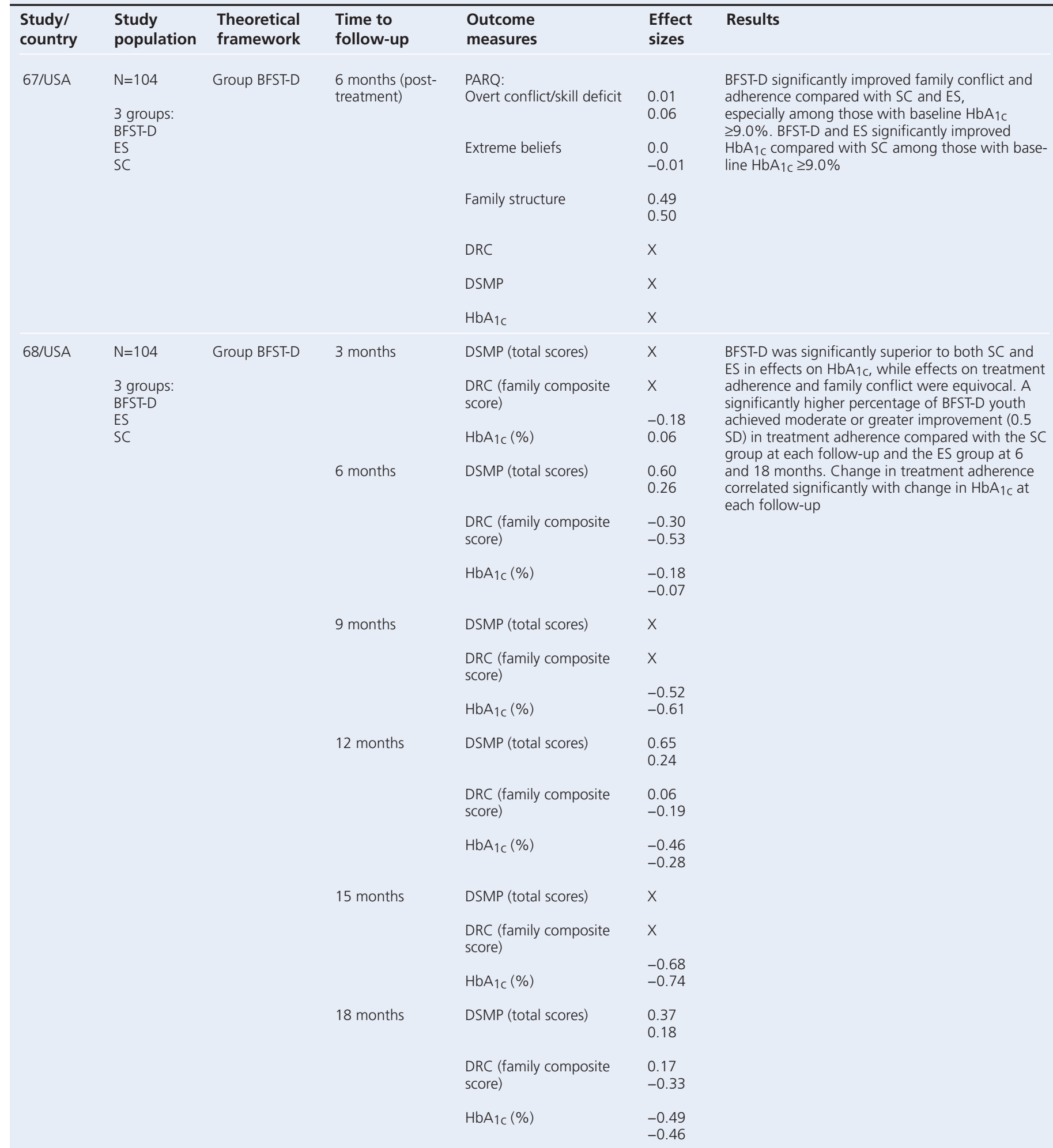


Table 2 Data extraction continued

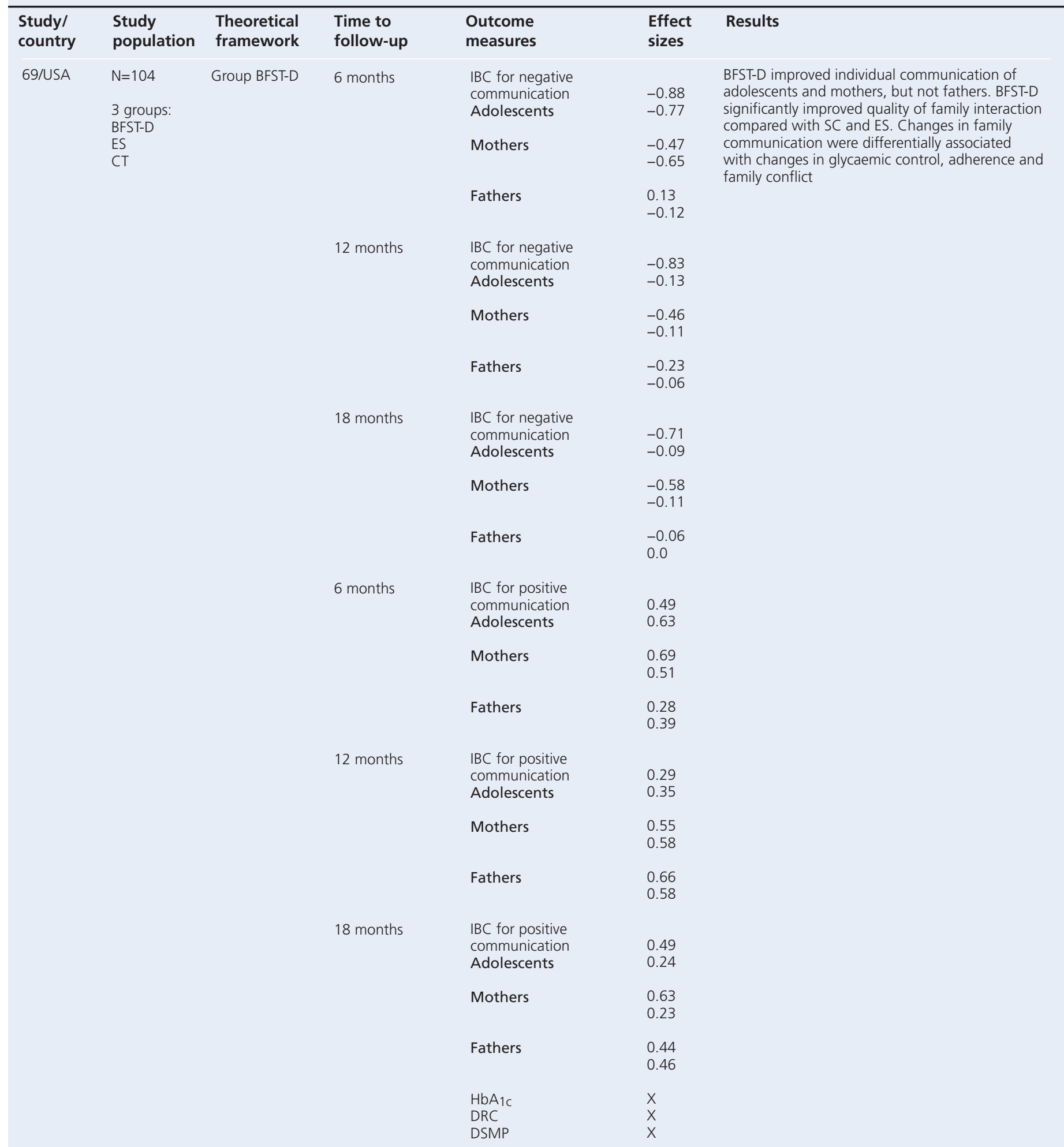


Table 2 Data extraction continued

\begin{tabular}{|c|c|c|c|c|c|c|}
\hline $\begin{array}{l}\text { Study/ } \\
\text { country }\end{array}$ & $\begin{array}{l}\text { Study } \\
\text { population }\end{array}$ & $\begin{array}{l}\text { Theoretical } \\
\text { framework }\end{array}$ & $\begin{array}{l}\text { Time to } \\
\text { follow-up }\end{array}$ & $\begin{array}{l}\text { Outcome } \\
\text { measures }\end{array}$ & $\begin{array}{l}\text { Effect } \\
\text { sizes }\end{array}$ & Results \\
\hline $63 /$ Sweden & $\begin{array}{l}\mathrm{N}=55 \\
2 \text { groups: } \\
\text { Intervention } \\
\text { Control }\end{array}$ & $\begin{array}{l}\text { Empowerment } \\
\text { theory }\end{array}$ & $\begin{array}{l}6 \text { months } \\
12 \text { months } \\
2 \text { months }\end{array}$ & $\begin{array}{l}\mathrm{HbA}_{1 \mathrm{c}} \\
\text { Empowerment scale }\end{array}$ & $\begin{array}{l}x \\
x\end{array}$ & 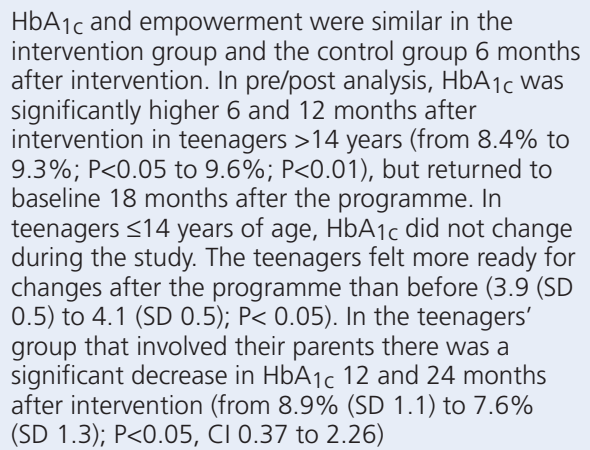 \\
\hline 72/USA & $\begin{array}{l}\mathrm{N}=30 \\
2 \text { groups: } \\
\text { Intervention } \\
\text { Control }\end{array}$ & $\begin{array}{l}\text { Peer group } \\
\text { and family-based }\end{array}$ & 6 months & $\begin{array}{l}\text { Primary care giver: } \\
\text { Brief symptom inventory } \\
\text { Behavioural assessment } \\
\text { scale for children } \\
\text { Diabetes family } \\
\text { relationship } \\
\text { questionnaire } \\
\text { Paediatric quality of life } \\
\text { inventory generic } \\
\text { Paediatric quality of life } \\
\text { family impact module } \\
\text { Readiness to change the } \\
\text { balance of responsibility } \\
\text { scale } \\
\text { SCl } \\
\text { Diabetes family } \\
\text { responsibility } \\
\text { questionnaire } \\
\text { Paediatric quality of life } \\
\text { inventory diabetes } \\
\text { Youth: } \\
\text { Brief symptom inventory } \\
\text { Diabetes family } \\
\text { relationship } \\
\text { questionnaire } \\
\text { Readiness to change the } \\
\text { balance of responsibility } \\
\text { scale }\end{array}$ & $\begin{array}{l}0.11 \\
0.47 \\
0.43 \\
0.00 \\
0.28 \\
0.09\end{array}$ & $\begin{array}{l}\text { At } 4 \text { months post treatment, parents and youths } \\
\text { reported increased parent responsibility, and } \\
\text { parents reported improved youth diabetes-specific } \\
\text { quality of life. Although there were no statistically } \\
\text { significant changes in } \mathrm{Hb}_{1 \mathrm{c}} \text { values and healthcare } \\
\text { utilisation frequency from } 6 \text { months prior to and } 6 \\
\text { months post treatment, other psychosocial changes } \\
\text { (ie, increases in parent responsibility and diabetes- } \\
\text { specific quality of life) were documented. } \\
\text { Therefore, this treatment was found to be a } \\
\text { promising intervention for use in an outpatient } \\
\text { clinical setting to aid in improving the psychosocial } \\
\text { functioning of youth with type } 1 \text { diabetes mellitus }\end{array}$ \\
\hline
\end{tabular}


Table 2 Data extraction continued

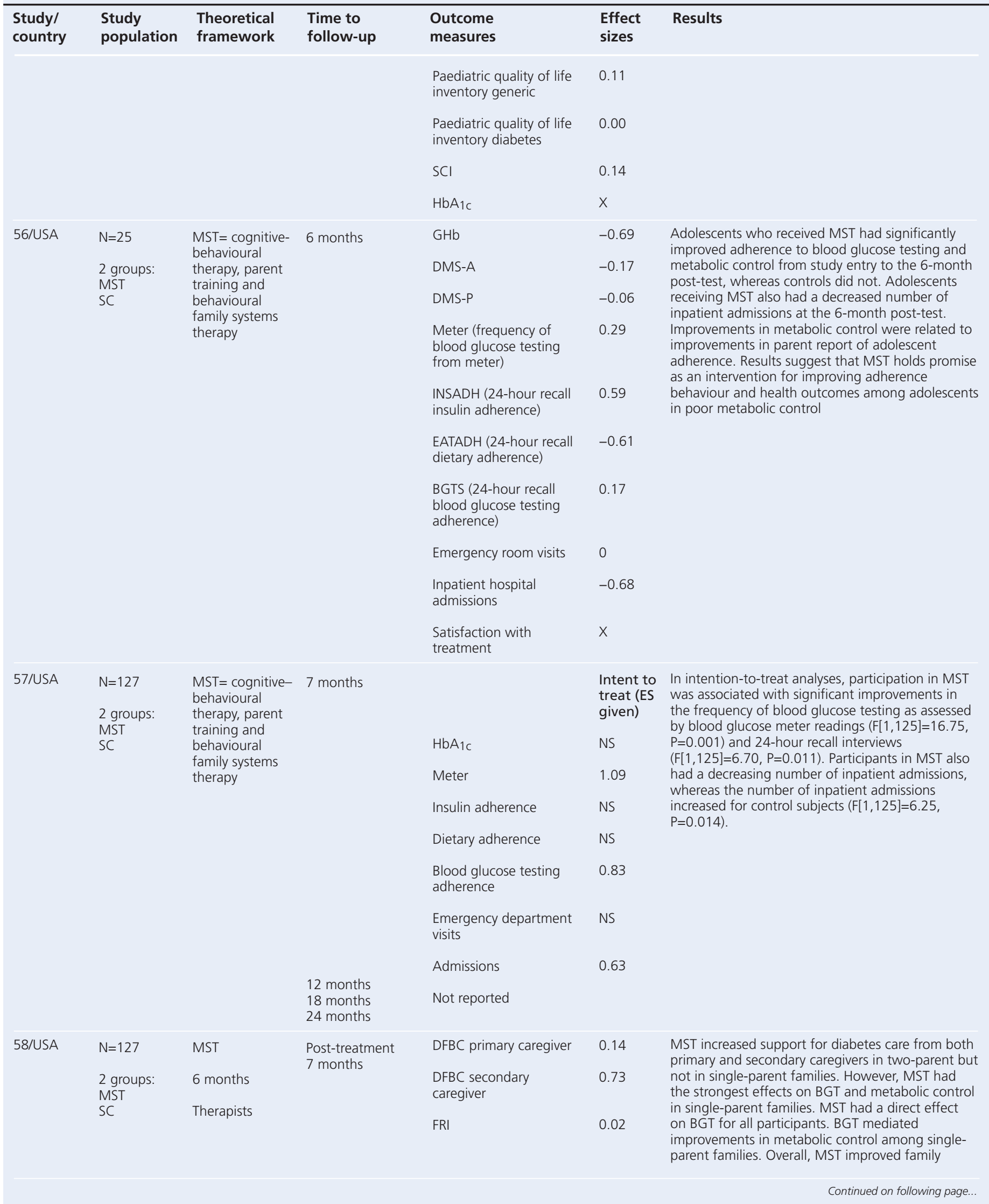


Table 2 Data extraction continued

\begin{tabular}{|c|c|c|c|c|c|c|}
\hline $\begin{array}{l}\text { Study/ } \\
\text { country }\end{array}$ & $\begin{array}{l}\text { Study } \\
\text { population }\end{array}$ & $\begin{array}{l}\text { Theoretical } \\
\text { framework }\end{array}$ & $\begin{array}{l}\text { Time to } \\
\text { follow-up }\end{array}$ & $\begin{array}{l}\text { Outcome } \\
\text { measures }\end{array}$ & $\begin{array}{l}\text { Effect } \\
\text { sizes }\end{array}$ & Results \\
\hline & & & $\begin{array}{l}12 \text { months } \\
18 \text { months } \\
24 \text { months } \\
\text { (not reported) }\end{array}$ & $\begin{array}{l}\mathrm{HbA}_{1 \mathrm{c}} \\
\mathrm{BGT} \text { frequency }\end{array}$ & $\begin{array}{l}-0.46 \\
0.73\end{array}$ & $\begin{array}{l}\text { relationships for youths with diabetes in two-parent } \\
\text { but not in single-parent families. Objective } \\
\text { outcomes related to diabetes were strongest for } \\
\text { single-parent families }\end{array}$ \\
\hline 61/USA & $\begin{array}{l}\mathrm{N}=70 \\
2 \text { groups: } \\
\text { Intervention } \\
\text { Control }\end{array}$ & $\begin{array}{l}\text { Social cognitive } \\
\text { theory, self- } \\
\text { regulation } \\
\text { models and } \\
\text { systems theory }\end{array}$ & $\begin{array}{l}6 \text { months } \\
12 \text { months } \\
18 \text { months } \\
24 \text { months }\end{array}$ & $\begin{array}{l}\mathrm{HbA}_{1 \mathrm{c}} \\
\mathrm{HbA}_{1 \mathrm{c}} \\
\mathrm{HbA}_{1 \mathrm{c}} \\
\mathrm{HbA}_{1 \mathrm{c}} \\
\text { Adherence in parents } \\
\text { and older youth 11+ } \\
\text { (DSMP) } \\
\mathrm{HbA}_{1 \mathrm{c}} \text { (age 12-14 only) } \\
\mathrm{HbA}_{1 \mathrm{c}} \text { (age 12-14 only) } \\
\mathrm{HbA}_{1 \mathrm{c}} \text { (age 12-14 only) } \\
\mathrm{HbA}_{1 \mathrm{c}} \text { (age 12-14 only) }\end{array}$ & $\begin{array}{l}x \\
x \\
x \\
x \\
x\end{array}$ & $\begin{array}{l}\text { A significant overall intervention effect on change } \\
\text { in glycemic control from baseline was observed at } \\
\text { the } 24 \text {-month interval ( } P=0.03 \text { ). Among } \\
\text { participants aged } 12-14 \text {, a significant effect on } \\
\text { glycaemic control was observed ( } P=0.009 \text { for } \\
\text { change from baseline to } 24 \text {-month interval; } \\
\mathrm{P}=0.035 \text { for mixed-effect model across study } \\
\text { duration). There was no intervention effect on } \\
\text { child or parent report of adherence; however, } \\
\text { associations of change in adherence with change in } \\
\text { glycemic control were weak. This clinic-integrated } \\
\text { behavioral intervention was effective in preventing } \\
\text { the deterioration in glycaemic control evident } \\
\text { during adolescence, offering a potential model for } \\
\text { integrating medical and behavioural sciences in } \\
\text { clinical care }\end{array}$ \\
\hline 60/USA & $\begin{array}{l}\mathrm{N}=32 \\
3 \text { groups: } \\
\mathrm{MF} \\
\mathrm{MF}+\mathrm{S} \\
\text { Control }\end{array}$ & $\begin{array}{l}\text { Group } \\
\text { multifamily } \\
6 \text { weeks } \\
\text { Psychological } \\
\text { social worker } \\
\text { and a nurse } \\
\text { practitioner }\end{array}$ & $\begin{array}{l}6 \text { weeks post- } \\
\text { intervention } \\
3 \text { months (planned } \\
\text { comparison) } \\
6 \text { months }\end{array}$ & $\begin{array}{l}\mathrm{HbA}_{1 \mathrm{c}} \\
\mathrm{HbA}_{1 \mathrm{c}} \\
\text { 2nd and 3rd study cycles } \\
\text { (with smaller group size) } \\
\mathrm{HbA}_{1 \mathrm{c}} \\
\mathrm{HbA}_{1 \mathrm{c}} \\
\text { Attitudes/perceptions }\end{array}$ & $\begin{array}{l}-0.63 \\
0.24 \\
-0.67 \\
0.27 \\
\\
\\
-0.02 \\
1.33 \\
0.10 \\
2.23 \\
x\end{array}$ & $\begin{array}{l}\text { Adolescents in the } \mathrm{MF}+\mathrm{S} \text { group displayed } \\
\text { significant decrements in HbA1c and adolescents in } \\
\text { both intervention groups reported more positive } \\
\text { perceptions of a 'teenager with diabetes' post- } \\
\text { treatment relative to controls. Adolescents } \\
\text { participating in smaller family groups demonstrated } \\
\text { clinically significant improvements in HbA1c that } \\
\text { were maintained at } 6 \text {-month follow-up. Parent } \\
\text { reports suggested that adolescents in the } \\
\text { intervention groups improved their diabetes care. } \\
\text { Findings support the use of multifamily groups plus } \\
\text { parent simulation of diabetes as an intervention } \\
\text { strategy for adolescents with type } 1 \text { diabetes }\end{array}$ \\
\hline $62 /$ USA & $\begin{array}{l}\mathrm{N}=21 \\
2 \text { groups: } \\
\text { Intervention } \\
\text { Control }\end{array}$ & $\begin{array}{l}\text { Social learning } \\
\text { theory } \\
3 \text { weeks } \\
\text { Psychology } \\
\text { graduate } \\
\text { student, } \\
\text { endocrinologist, } \\
\text { ophthalmologist, } \\
\text { podiatrist }\end{array}$ & 4 months & $\begin{array}{l}\mathrm{HbA}_{1 \mathrm{c}} \\
\text { Diabetes knowledge } \\
\text { Attitude } \\
\text { Behaviour, Sarason social } \\
\text { support questionnaire } \\
\text { MEPS test }\end{array}$ & $x$ & $\begin{array}{l}\text { Four months after the intervention, } \mathrm{HbA}_{1 \mathrm{c}} \text { was } \\
\text { significantly lower in the social skills intervention } \\
\text { group. A variety of variables were significantly } \\
\text { correlated with good metabolic control. These } \\
\text { included self-reported compliance with a diabetes } \\
\text { regimen and attitudes toward self-care. } \\
\text { Unexpectedly, variables correlated with poor } \\
\text { diabetes control included social problem-solving } \\
\text { ability and satisfaction with social support }\end{array}$ \\
\hline 59/USA & $\begin{array}{l}\mathrm{N}=58 \\
2 \text { groups: } \\
\text { BFST } \\
\text { Control }\end{array}$ & BFST-D model & $\begin{array}{l}\text { Post-treatment } \\
\text { (6-10 weeks) }\end{array}$ & $\begin{array}{l}\text { DRC } \\
\text { Adolescents } \\
\text { Mothers } \\
\text { Fathers }\end{array}$ & $\begin{array}{l}-0.97 \\
-1.27 \\
-1.01\end{array}$ & $\begin{array}{l}\text { Home-based BFST produced change in diabetes- } \\
\text { related family conflict ranging from } 1 / 3 \text { to } 1 / 2 \text { SD } \\
\text { and general family conflict ranging from } 1 / 3 \text { to } 3 / 4 \\
\text { SD }\end{array}$ \\
\hline
\end{tabular}


Table 2 Data extraction continued

\begin{tabular}{|c|c|c|c|c|c|c|}
\hline $\begin{array}{l}\text { Study/ } \\
\text { country }\end{array}$ & $\begin{array}{l}\text { Study } \\
\text { population }\end{array}$ & $\begin{array}{l}\text { Theoretical } \\
\text { framework }\end{array}$ & $\begin{array}{l}\text { Time to } \\
\text { follow-up }\end{array}$ & $\begin{array}{l}\text { Outcome } \\
\text { measures }\end{array}$ & $\begin{array}{l}\text { Effect } \\
\text { sizes }\end{array}$ & Results \\
\hline & & & & $\begin{array}{l}\text { CBQ } \\
\text { Adolescents } \\
\text { Mothers } \\
\text { Fathers } \\
\mathrm{HbA}_{1 \mathrm{c}}\end{array}$ & $\begin{array}{l}-0.41 \\
-0.39 \\
-0.07 \\
x\end{array}$ & \\
\hline 71/USA & $\begin{array}{l}\mathrm{N}=39 \\
2 \text { groups: } \\
\text { Intervention } \\
\text { Control }\end{array}$ & $\begin{array}{l}\text { Positive } \\
\text { psychology }\end{array}$ & 3 and 6 months & $\begin{array}{l}\text { Positive and negative } \\
\text { affect scale } \\
\text { Children's depression } \\
\text { inventory } \\
\mathrm{SCl} \\
\text { Diabetes family conflict } \\
\text { scale } \\
\text { Quality of life inventory } \\
\text { Mean frequency of } \\
\text { blood glucose } \\
\text { monitoring } \\
\mathrm{HbA} 1 \mathrm{c}\end{array}$ & $x$ & $\begin{array}{l}\text { No main effects for treatment were observed at } \\
\text { the } 6 \text {-month follow-up. However, there was a } \\
\text { significant association between adolescents' levels } \\
\text { of positive affect and measures of adherence, } \\
\text { including self-report and meter downloads of } \\
\text { glucose monitoring }\end{array}$ \\
\hline
\end{tabular}

The results indicated that adolescents and their mothers who had experienced BFST rated it as significantly more acceptable, applicable and effective in terms of improving family communication, problem-solving and conflict-resolution skills than did those who experienced the ES intervention. Furthermore, Wysocki and his associates $^{68}$ demonstrated that 10 sessions of BFST improved family communication and problem-solving based on parent and adolescent reports ${ }^{65}$ and direct observation of family interactions. ${ }^{73}$ These benefits persisted for 12 months. ${ }^{66}$ Wysocki and co-workers conducted another randomised controlled trial implementing a modified version of BFST-D.67,68 The results demonstrated significant improvements in glycaemic control, treatment adherence and diabetes-related family conflict immediately post-treatment. These effects were maintained over a 12-month follow-up period. ${ }^{69}$

\section{Theory of social ecology underlying Multi-Systemic Therapy} (families and adolescents)

This theory has been used as a conceptual framework of MultiSystemic Therapy (MST; see Box 1). MST is an intensive home-based treatment model, designed to target adherence-related problems within the family system, schools, peer network, healthcare system and broader community systems. Ellis and colleagues ${ }^{56}$ demonstrated that adolescents who received MST had significantly im- proved adherence to blood glucose testing and metabolic control (compared with standard care) at 6 months post-test. In general, parents in the treatment condition reported high levels of satisfaction with MST. Adolescents who received MST had a significantly decreased number of hospital admissions during the 6-month study period.

Following the findings of this study, Ellis and colleagues conducted a study in 2005, which demonstrated that participation in MST was associated with significant improvements in the frequency of blood glucose testing and 24-hour recall interviews. ${ }^{57}$ The MST group also had a decreased number of inpatient admissions whereas the number of inpatient admissions increased among control group participants. Furthermore, the findings of their study in 2007 demonstrated that MST increased support for diabetes care from both primary and secondary caregivers in two-parent but not in single-parent families. However, MST had the strongest effects on blood glucose testing and metabolic control in single-parent families.

Following the review of the MST and BFST, Kichler et a ${ }^{72}$ developed the 'Kicking in Diabetes Support' project, which aimed to provide both peer group and family-based interventions to adolescents with type 1 diabetes and their parents. Although there were no statistically significant changes in $\mathrm{HbA}_{1 \mathrm{c}}$ and healthcare utilisation 
frequency at 6 months post-treatment, other psychosocial changes were documented, such as increases in parent responsibility and parents reported improved youth diabetes-specific quality of life. Therefore, this intervention appears to be a treatment modality that is feasible, acceptable and adaptable in a clinical setting by licensed psychologists and trainees (to improve the psychosocial functioning of youths with type 1 diabetes). ${ }^{72}$

\section{Family systems theory underlying multi-family group intervention plus parent simulation (parents and adolescents)} Family systems approach is applied within a group therapy format (mentioned above). One study ${ }^{60}$ evaluated the impact of a 6-week multi-family (MF) group intervention and parent simulation (S) on adolescents' metabolic control and psychosocial and family functioning. The results demonstrated significant improvements/decrements in metabolic control in the MF and S groups in comparison with the control group in the first, second and third cycle of the study. In terms of perception/attitude, improvement was noted in adolescents' attitudes towards a teenager with diabetes for those in the MF and MF+S groups relative to the controls. Overall, it is important for parents and their child to gain a better insight into each other's views. Asking parents to simulate diabetes management for 1 week was a novel way of trying to achieve this. ${ }^{74}$

Please see the supplementary material in Appendix C (see www.bjd-abcd.com) for a brief description about effect sizes.

\section{Discussion}

Seventeen studies were included in this review, but few studies included the same comparisons and outcomes and therefore a metaanalysis was not conducted. The initial studies by Wysocki and colleagues ${ }^{65,66}$ reported that BFST compared with current therapy and ES yielded more improvements in parent/adolescent relationships. However, in 2006 they reported non-significant findings related to the same measure (all subscales of the Parent-Adolescent Relationship Questionnaire $=$ NS). ${ }^{67}$ In 2000 it was indicated that their intervention had no effect on treatment adherence at posttreatment. ${ }^{65}$ This was contrary to the results reported by them in 2001 , in which the BFST group showed significantly improved treatment adherence at 6 and 12 months follow-up. ${ }^{66}$ In their 2000 study Wysocki et al included 3-month follow-up but, unlike other studies, they did not measure outcomes at 6 and 12 months. ${ }^{65}$ It may be that short-term behavioural approach-based interventions promote improvements in parent/adolescent relationships. However, treatment adherence can be improved and maintained by a prolonged change in family interaction patterns.

Although Viklund et al tailored their intervention to support adolescents through the empowerment programme, no positive glycaemic or empowerment effects were found for teenagers with diabetes. ${ }^{63}$ One interpretation was that perhaps the teenagers $(<14$ years) were too young to incorporate the described components of empowerment. ${ }^{75}$ Additionally, among teenagers who invited their parents to participate in the intervention, $\mathrm{HbA}_{1 \mathrm{c}}$ did not increase, which was in accordance with the literature, suggesting the importance of family support to facilitate coping with everyday management and demands of diabetes. $6,76,77$
In this review, a variety of interventions which resulted in significant reductions in $\mathrm{HbA}_{1} c^{56,57,67,68}$ involved parents and had beneficial effects on diabetes management. The parental simulation approach used by Satin and colleagues ${ }^{60}$ also resulted in a large beneficial effect on $\mathrm{HbA}_{1}$. Research has suggested that the frequency of blood glucose monitoring is increased with parental involvement which, in turn, is associated with better metabolic control. ${ }^{78}$ Furthermore, a high level of support from family members leads adolescents to better adhere to their diabetes regimen. ${ }^{14,79}$

A variety of other interventions indicate that family-based behavioural approaches such as goal-setting, self-monitoring, positive reinforcement, behavioural contracts, supportive parental communications and appropriately shared responsibility for diabetes management have improved regimen adherence, parent/adolescent relationships and glycaemic control. 6,80 However, low levels of family support and increased family conflict have been consistently associated with poor diabetes self-management, metabolic control, psychosocial adaptation and quality of life in adolescents with type 1 diabetes. $2,11,81$

On the other hand, peer group interventions indicate that peer group support and problem solving can improve short-term glycaemic control. 44,62 Group coping skill training improved glycaemic control and quality of life for adolescents involved in intensive insulin regimens. ${ }^{82}$ Stress management, problem solving and coping skill training, delivered in small groups, has reduced diabetes-related stress, ${ }^{83}$ improved social interaction,, 84 increased glucose monitoring and improved glycaemic control. ${ }^{85}$

In the current review, the importance of integrating medical, behavioural and psychosocial components into the interventions were underscored by at least six of the RCTs.56,67-69,71-72 The results suggest that multi-component interventions may be more successful for adolescents than those that just focus on one aspect - for example, psychological in the study by Newton and Ashley. ${ }^{70}$

Home-based interventions, although more time consuming for healthcare professionals (HCPs), appear to be a viable and accessible alternative for intervening with many of the families. ${ }^{56}$ Such interventions provide an ecologically valid family-centered means of engaging adolescents and their parents in treatments. ${ }^{86}$ In addition, they may also allow the HCPs to better understand the 'real-world' barriers to regimen adherence. It is also important to note that the home-based intervention by Ellis et a ${ }^{57}$ demonstrated high rates of recruitment (70\%) as well as high retention rates $(75 \%)$ in a treatment that lasted $>5$ months on average. This suggests that, when such interventions are provided in a way that increases access, they have a high likelihood of being accepted by such adolescents and their families. ${ }^{57}$ Cost-effectiveness studies are needed to assess whether the greater uptake and effects offset the costs of staff travel.

\section{Strengths of the study}

This review was not limited to one geographical area or one country. Furthermore, to the best of our knowledge, no systematic review has evaluated family/peer- and theory-based interventions involving adolescents and families/peers. Studies included in the review were RCTs, which reduced evaluation of 
interventions with recruitment and other study design bias eliminated by randomisation.

\section{Limitations of the study}

It is acknowledged that this review includes a relatively small number of studies with rather small sample sizes, which makes it difficult to draw conclusions from the results. Interventions developed in the USA require modification and re-evaluation for application in the UK. ${ }^{87}$ This review exposed interventions, mostly including parents and rarely including peers, which were evaluated by assessing widely differing outcomes. Due to the heterogeneity of the studies, it was not possible to perform a meta-analysis.

The heterogeneity of young people with diabetes was generally not reflected in the RCTs undertaken to date, although some did select those with poor glucose control. A patient group at high risk are those with repeated hospitalisations, who are particularly likely to have a high prevalence of psychosocial issues and among whom a combined outreach diabetes specialist-mental health team approach might be able to reduce admissions and length of stay and improve glycaemic control. 88

\section{Conclusion}

Overall, interventions including parents have small- to large-sized beneficial effects on a variety of diabetes management outcomes. Studies identified here addressing the issue of parental involvement support the evidence that developmentally appropriate and negotiated responsibility has beneficial outcomes. Narrative analysis suggests that interventions are more likely to be effective if they demonstrate the inter-relatedness of the various aspects of diabetes management. The evaluation of interventions needs to be by welldesigned theory-based RCTs, of sufficient size, over a long-term period, which report results in such a way that effect sizes can be calculated. Home-based interventions could be more viable and accessible alternatives for intervening with many of the families than office- or hospital-based interventions. Future studies should estimate the costs associated with delivering such interventions involving families and also clarify the theoretically guided design of the intervention and the selection of the outcomes. The development process should involve stakeholders (ie, adolescents, their families and HCPs) to co-design a potentially cost-effective and feasible intervention in the context of NHS diabetes services. Such interventions need to be understood and accepted by the HCPs and managers as key and integral parts of diabetes care.

\section{Conflict of interest None. Funding None.}

\section{References}

1. Glasgow RE, Fisher EB, Anderson BJ, et al. Behavioural science in diabetes: contributions and opportunities. Diabetes Care1999;22:832-43. https://doi.org/10.2337/diacare.22.5.832

2. Pendley JS, Kasmen LJ, Miller DL, et al. Peer and family support in children and adolescents with T1D. J Pediatr Psychol 2002;27:429-38. https://doi.org/10.1093/jpepsy/27.5.429

3. Diabetes Control and Complications Trial. The effect of intensive treatment of diabetes on the development and progression of long-term complications in insulin dependent diabetes mellitus. N Engl J Med 1993;329:977-86. https://doi.org/10.1056/NEJM199309303291401

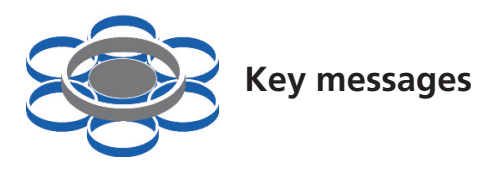

- Interventions involving parents had beneficial effects on diabetes management and significant reduction in $\mathrm{HbA}_{1 \mathrm{c}}$

- Family-based behavioural approaches such as goalsetting, self-monitoring, positive reinforcement, behavioural contracts, supportive parental communications, and appropriately shared responsibility for diabetes management showed improved regimen adherence, parent-adolescent relationship and glycaemic control

- Multi-component interventions may be more successful for adolescents than those just focusing upon one aspect

4. Diabetes Control and Complications Trial. Effect of intensive diabetes treatment on the development of long-term complications in adolescents with insulin-dependent diabetes mellitus. J Pediatr 1994;125:17788. https://doi.org/10.1016/S0022-3476(94)70190-3

5. Delamater AM. Psychological care of children and adolescents with diabetes. Pediatr Diabetes 2009;10:175-84. http://dx.doi.org/10.1111/j.1399-5448.2009.00580.x

6. Anderson BJ, Brackett BA, Joyce $H$, et al. An office-based intervention to maintain parent-adolescent teamwork in diabetes management. Diabetes Care1999;22:713-21. https://doi.org/10.2337/diacare.22.5.713

7. Laffel LM, Vangsness L, Connell A, et al. Impact of ambulatory, familyfocused teamwork intervention on glycaemic control in youth with T1D. J Pediatr 2003;142:409-16. http://dx.doi.org/10.1067/mpd.2003.138

8. Berg CA, Wiebe DJ, Beveridge RM, et al. Mother child appraisal involvement in coping with diabetes stressors and emotional adjustment. J Pediatr Psycho/ 2007;32:995-1005. http://dx.doi.org/10.1093/jpepsy/ jsm043

9. Cameron F, Skinner T, De Beaufort C, et al. Hvidoere Study Group on Childhood Diabetes. Are family factors universally related to metabolic outcomes in adolescents with T1D? Diabet Med 2008;25:463-8. https://doi.org/10.1111/j.1464-5491.2008.02399.x

10. Schilling LS, Knafl KA, Grey M. Changing patterns of self-management in youth with type I diabetes. J Pediatr Nurs 2006;21:412-24. http://dx.doi.org/10.1016/j.pedn.2006.01.034

11. Wysocki T. Associations among teen-parent relationships, metabolic control, and adjustment to diabetes in adolescents. J Pediatr Psychol 1993;18:441-52. https://doi.org/10.1093/jpepsy/18.4.441

12. Wysocki T, Taylor A, Hough BS, et al. Deviation from developmentally appropriate self-care autonomy: association with diabetes outcomes. Diabetes Care 1996;19:119-25. https://doi.org/10.2337/diacare.19.2.119

13. Greco P, Greca AM, Auslander W, et al. Family and peer support of diabetes care among adolescents. Diabetes 1991;40:537A.

14. La Greca AM, Auslander WF, Greco P, et al. I get by with a little help from my family and friends: adolescents' support for diabetes care. J Pediatr Psychol 1995;20:449-76. https://doi.org/10.1093/jpepsy/20.4.449

15. Greco P, Pendley JS, McDonell K, Reeves G. A peer group intervention for adolescents with T1D and their best friends. J Pediatr Psychol 2001;26:485-90. https://doi.org/10.1093/jpepsy/26.8.485

16. Fuligni AJ, Eccles JS, Barber BL, Clements P. Early adolescent peer orientation and adjustment during high school. Devel Psychol 2001;37:2836. https://doi.org/10.1037/0012-1649.37.1.28

17. Harvey JN. Health beliefs and health outcomes. In: Martin LR, DiMatteo MR, eds. The Oxford Handbook of Health Communication, Behaviour Change and Treatment Adherence. New York: Oxford University Press, 2013:17792. https://doi.org/10.1093/oxfordhb/ 9780199795833.013.006

18. Diefenebach MA, Leventhal $H$. The common-sense model of illness representation: theoretical and practical considerations. J Social Distress 
Homeless 1996;5:11-38. https://doi.org/10.1007/BF02090456

19. Leventhal H, Hudson S, Robitaille C. Health, coping, and well-being perspectives from social comparison theory. In: Buunk BP, Gibbons FX, eds. Social Comparison and Health: A Process Model. Mahweh, NJ: Lawrence Erlbaum Associates, 1997

20. Browning KK, Wewers ME, Ferketich AK, Otterson GA, Reynolds NR The Self-regulation Model of Illness applied to smoking behaviour in lung cancer. Cancer Nursing 2009:32:E15-25. https://doi.org/10.1097/NCC.0b013e3181a0238f

21. Henderson DJ. Consciousness-rising as a feminist nursing action. Promise and practice, present and future. In: Thorne SE, Hayes VE, eds. Nursing Praxis: Knowledge and Action. London: Sage Publications, 1997

22. Laverack G. Empowerment and Professional Practice. Basingstoke: Palgrave Macmillan, 2005

23. Gibson $\mathrm{CH}$. The process of empowerment in mothers of chronically ill children. J Advan Nursing 1995;21:1201-10. https://doi.org/10.1046/j.1365-2648.1995.21061201.x

24. Naidoo J, Wills J. Health Promotion: Foundations for Practice. London: Elsevier Health Sciences, 2000.

25. Craig PM, Lindsay GM. Nursing for Public Health: Population-Based Care. New York: Elsevier Health Sciences, 2000.

26. Anderson R, Funnell M. Compliance and adherence are dysfunctional concepts in diabetes care. Diabetes Educ 2000;26:597-604. https://doi.org/10.1177/014572170002600405

27. Tol A, Alhani F, Shojaeazadeh D, et al. An empowering approach to promote the quality of life and self-management among type 2 diabetic patients. J Educ Health Promot 2015:4:13. http://dx.doi.org/10.4103/2277-9531.154022

28. Williams BW, Kessler HA, Williams MV. Relationship among practice change, motivation, and self-efficacy. J Contin Educ Health Prof 2014; 34(Suppl 1):S5-S10.7. http://dx.doi.org/10.1002/chp.21235

29. Bandura A. Self-efficacy: toward a unifying theory of behaviour change. Psychol Rev 1977:84:191-215. https://doi.org/10.1037/0033-295X.84.2.191

30. Dehghan H, Charkazi A, Mohammad Kouchaki G, et al. General selfefficacy and diabetes management self-efficacy of diabetic patients referred to diabetes clinic of Aq Qala, North of Iran. J Diabetes Metab Disord 2017:16:8. http://dx.doi.org/10.1186/s40200-016-0285-z

31. Bandura A. Social cognitive theory of self-regulation. Organizational Behaviour and Human Decision Processes 1991;50:248-87. https://doi.org/10.1016/0749-5978(91)90022-L.

32. Bandura A. Social Foundations of Thought and Action: A Social Cognitive Theory. Englewood Cliffs, NJ: Prentice-Hall,1986.

33. Yi-Frazier JP, Hilliard M, Cochrane K, Hood KK. The impact of positive psychology on diabetes outcomes: a review. Psychology 2012; 3(12A):1116-24. http://dx.doi.org/10.4236/psych.2012.312A165

34. Bronfenbrenner U. The Ecology of Human Development: Experiments by Design and Nature. Cambridge, MA: Harvard University Press, 1979.

35. Minuchin S. Families and Family Therapy. Cambridge, MA: Harvard University Press, 1974

36. Haley J. Problem-Solving Therapy. 2nd edn. San Franciso: Jossey-Bass, 1987.

37. Henggeler SW, Sheidow AJ. Empirically supported family-based treatments for conduct disorder and delinquency in adolescents. J Marital Fam Ther 2012;38:30-58. http://dx.doi.org/10.1111/j.1752-0606.2011.00244.x

38. Gordon SB, Davidson N. Behavioural parent training. In: Gurman AS, Kniskern DP, eds. Handbook of Family Therapy. New York: Brunner/Mazel, 1981

39. Jacobson NS, Margolin G. Marital Therapy: Strategies Based on Social Learning and Behaviour Exchange Principles. New York: Brunner/Mazel, 1979.

40. Patterson GR. Coercive Family Process. Eugene, OR: Castalia, 1982.

41. Aponte HJ, VanDeusen JM. Structural family therapy. In: Gurman AS, Kniskern DP, eds. Handbook of Family Therapy. New York: Brunner/Mazel, 1981.

42. Steinglass P. A systems view of family interaction and psychopathology. In: Jacob T, ed. Family Interaction and Psychopathology. New York: Plenum Press, 1987. https://doi.org/10.1007/978-1-4899-0840-7_2

43. Robin AL, Foster SL. Negotiating Parent-Adolescent Conflict: A Behavioural-Family System Approach. New York, London: Guilford Press, 1989
44. Anderson BJ, Wolf FM, Burkhart MT, et al. Effects of a peer group intervention on metabolic control of adolescents with IDDM: randomized outpatient study. Diabetes Care 1989;12:184-8. https://doi.org/10.2337/diacare.12.3.179

45. Løding RN, Wold JE, Skavhaug Å. Experiences with a group intervention for adolescents with T1D and their parents. Eur Diabetes Nursing 2008;5:9-14. https://doi.org/10.1002/edn.96

46. Hansson K, Ryden O, Johnsson P. Parent-related family climate: a concomitant to metabolic control in juvenile IDDM. Fam Syst Med 1994;12:405-13. https://doi.org/10.1037/h0089272

47. Løding RN, Wold JE, Skavhaug Å, Graue M. Evaluation of peer-group support and problem-solving training in the treatment of adolescents with T1D. Eur Diabetes Nursing 2007;4:28-33. https://doi.org/10.1002/edn.73

48. Salamon KS, Hains AA, Fleischman KM, Davies WH, Kichler J. Improving adherence in social situations for adolescents with type 1 diabetes mellitus (T1DM): a pilot study. Primary Care Diabetes 2010;4:47-55. http://dx.doi.org/10.1016/j.pcd.2009.10.003

49. Harris MA, Harris BS, Mertlich D. Brief report: in-home family therapy for adolescents with poorly controlled diabetes: failure to maintain benefits at 6-month follow-up. J Pediatr Psychol 2005;30:683-8. http://dx.doi.org/10.1093/jpepsy/jsi055

50. Ellis DA, Naar-King S, Templin T, et al. Multisystemic therapy for adolescents with poorly controlled T1D: reduced diabetic ketoacidosis admissions and related costs over 24 months. Diabetes Care 2008:31:1746-7. http://dx.doi.org/10.2337/dc07-2094

51. Forsander GA, SundelinJ, Persson B. Influence of the initial management regimen and family social situation on glycaemic control and medical care in children with type 1 diabetes mellitus. Acta Paediatr 2000; 89:1462-8.

52. Nansel T, lonnotti RJ, Simons-Morton BG, et al. Long-term maintenance of treatment outcomes: diabetes personal trainer intervention for youth with type 1 diabetes. Diabetes Care 2009;32:807-09. http://dx.doi.org/10.2337/dc08-1968

53. Philis-Tsimikas A, Fortmann A, Lleva-Ocana L, Walker C, Gallo LC. Peerled diabetes education programs in high-risk Mexican Americans improve glycaemic control compared with standard approaches: a Project Dulce promotora randomized trial. Diabetes Care 2011;34:1926-31. http://dx.doi.org/10.2337/dc10-2081

54. Hampson SE, Skinner TC, Hart J, et al. Effects of educational and psychological interventions for adolescents with diabetes mellitus: a systematic review. Health Technol Assess 2001:5:1-77. https://doi.org/10.3310/hta5100

55. Thomas BH, Ciliska D, Dobbins M, Micucci S. A process for systematically reviewing the literature: providing the research evidence for public health nursing interventions. Worldviews Evid Based Nurs 2004:1:17684. http://dx.doi.org/10.1111/j.1524-475X.2004.04006.x

56. Ellis DA, Naar-King S, Frey $M$, et al. Use of multi-systemic therapy to improve regimen adherence among adolescents with T1D in poor metabolic control: a pilot investigation. J Clin Psychol Medical Settings 2004;11:315-24. https://doi.org/10.1023/B:JOCS.0000045351.98563.4d

57. Ellis DA, Frey M, Naar-King S, et al. Use of multisystemic therapy to improve regimen adherence among adolescents with type 1 diabetes in chronic poor metabolic control: a randomized controlled trial. Diabetes Care 2005;28:1604-10. https://doi.org/10.2337/diacare.28.7.1604

58. Ellis DA, Yopp J, Templin T, et al. Family mediators and moderators of treatment outcomes among youths with poorly controlled type 1 diabetes: results from a randomized controlled trial. J Pediatr Psychol 2007;32:194-205. http://dx.doi.org/10.1093/jpepsy/jsj1 16

59. Harris MA, Freeman KA, Beers M. Family therapy for adolescents with poorly controlled diabetes: initial test of clinical significance. J Pediatr Psychol 2009;34:1097-107. http://dx.doi.org/10.1093/jpepsy/jsp009

60. Satin W, La Greca AM, Zigo MA, Skyler JS. Diabetes in adolescence. Effects of multifamily group intervention and parent simulation of diabetes. J Pediatr Psychol 1989;14:259-75. https://doi.org/10.1093/jpepsy/14.2.259

61. Nansel TR, lannotti RJ, Liu A. Clinic-integrated behavioral intervention for families of youth with T1D: randomized clinical trial. Pediatrics 2012:129:e866-73. http://dx. doi.org/10.1542/peds.2011-2858

62. Kaplan RM, Chadwick MW, Schimmel LE. Social learning intervention 
to promote metabolic control in type I diabetes mellitus: pilot experiment results. Diabetes Care 1985:8:152-5.

https://doi.org/10.2337/diacare.8.2.152

63. Viklund G, Ortqvist E, Wikblad K. Assessment of an empowerment education programme. A randomized study in teenagers with diabetes. Diabet Med 2007;24:550-6. http://dx.doi.org/10.1111/j.1464-5491.2007.02114.x

64. Wysocki T, Harris M, Greco P, et al. Social validity of support group and behaviour therapy interventions for families of adolescents with insulindependent diabetes mellitus. J Pediatr Psychol 1997;22:635-49. https://doi.org/10.1093/jpepsy/22.5.635

65. Wysocki T, Harris MA, Greco P, et al. Randomized controlled trial of behaviour therapy for families of adolescents with insulin-dependent diabetes mellitus. J Pediatr Psycho/ 2000;25:23-33. https://doi.org/10.1093/jpepsy/25.1.23

66. Wysocki T, Greco P, Harris MA, et al. Behaviour therapy for families of adolescents with diabetes: maintenance of treatment effects. Diabetes Care 2001;24:441-6. https://doi.org/10.2337/diacare.24.3.441

67. Wysocki T, Harris MA, Buckloh LM, et al. Effects of behavioural family systems therapy for diabetes on adolescents' family relationships, treatment adherence, and metabolic control. J Pediatr Psychol 2006;31:92838. http://dx.doi.org/10.1093/jpepsy/jsj098

68. Wysocki T, Harris MA, Buckloh LM, et al. Randomized trial of behavioural family systems therapy for diabetes: maintenance of effects on diabetes outcomes in adolescents. Diabetes Care 2007;30:555-60. http://dx.doi.org/10.2337/dc06-1613

69. Wysocki T, Harris MA, Buckloh LM, et al. Randomized controlled trial of behavioural family systems therapy for diabetes: maintenance and generalization of effects on parent-adolescent communication. Behav Ther 2008;39:33-46. https://doi.org/10.1016/j.beth.2007.04.001

70. Newton KT, Ashley A. Pilot study of a web-based intervention for adolescents with type 1 diabetes. J Telemed Telecare 2013;19:443-9. http://dx.doi.org/10.1177/1357633X13512069

71. Jaser SS, Patel N, Linsky R, Whittemore R. Development of a positive psychology intervention to improve adherence in adolescents with type 1 diabetes. J Pediatr Health Care 2014;28:478-85. http://dx.doi.org/10.1016/j.pedhc.2014.02.008

72. Kichler JC, Kaugars AS, Marik P, Nabors L, Alemzadeh R. Effectiveness of groups for adolescents with type 1 diabetes mellitus and their parents. Families System Health 2013;31:280-93. https://doi.org/10.1037/a0033039

73. Wysocki T, Miller K, Greco P, et al. Behaviour therapy of families of adolescents with diabetes: effects on directly observed family interactions. Behav Ther 1999;30:507-25. https://doi.org/10.1016/S0005-7894(99)80022-7

74. Harvey JN. Psychosocial interventions for the diabetic patient. Diabetes Metab Syndr Obes 2015:8:29-43. http://dx.doi.org/10.2147/DMSO.S44352

75. Funnell MM, Anderson RM, Arnold MS, et al. Empowerment: an idea whose time has come in diabetes education. Diabetes Educ 1991; 17:37-41. https://doi.org/10.1177/014572179101700108

76. Graue M, Wentzel-Larsen T, Hanestad BR, Sovik O. Health-related quality of life and metabolic control in adolescents with diabetes: the role of parental care, control, and involvement. J Pediatr Nurs 2005;20:37382. http://dx.doi.org/10.1016/j.pedn.2005.08.005

77. Barlow JH, Ellard DR. Psycho-educational interventions for children with chronic disease, parents and siblings: an overview of the research evidence base. Child Care Health Devel 2004;30:637-45. https://doi.org/10.1111/j.1365-2214.2004.00474.x

78. Anderson B, Ho J, Brackett J, Finkelstein D, Laffel L. Parental involvement in diabetes management tasks: relationships to blood glucose monitoring adherence and metabolic control in young adolescents with insulindependent diabetes mellitus. J Pediatr 1997;130:257-65. https://doi.org/10.1016/S0022-3476(97)70352-4

79. Forsander GA, Persson B, Sundelin J, et al. Metabolic control in children with insulin-dependent diabetes mellitus 5 years after diagnosis: early detection of patients at risk for poor metabolic control. Acta Paediatr 1998;87:857-64. https://doi.org/10.1111/j.1651-2227.1998.tb01551.x

80. Murphy H, Rayman G, Skinner TC. Psycho-educational interventions for children and young people with type 1 diabetes. Diabet Med 2006;23:935-43. http://dx.doi.org/10.1111/j.1464-5491.2006.01816.x

81. Whittemore R, Kanner S, Grey M. The influence of family on physiological and psychosocial health in youth with type 1 diabetes: a systematic review. In: Melnyk B, Fineout-Overholt E, eds. Evidence-Based Practice in Nursing and Healthcare: A Guide to Best Practice. Philadelphia: Lippincott Williams \& Wilkins, 2004.

82. Boland EA, Grey M, Oesterle AL, Fredrickson L, Tamborlane WV. Continuous subcutaneous insulin infusion: a new way to lower risk of severe hypoglycaemia, improve metabolic control, and enhance coping in adolescents with type 1 diabetes. Diabetes Care 1999:22:1779-84. https://doi.org/10.2337/diacare.22.11.1779

83. Hains AA, Davies WH, Parton E, Totka J, Amoroso-Camarata J. A stress management intervention for adolescents with T1D. The Diabetes Educator 2000;26:417-24. https://doi.org/10.1177/014572170002600309

84. Mendez F, Belendez M. Effects of a behavioural intervention on treatment adherence and stress management in adolescents with IDDM. Diabetes Care 1997;20:1370-5. https://doi.org/10.2337/diacare.20.9.1370

85. Cook S, Herold K, Edidin DV, Briars R. Increasing problem solving in adolescents with type 1 diabetes: the Choices Diabetes Program. The Diabetes Educator 2002;28:115-24. https://doi.org/10.1177/014572170202800113

86. Harris MA, Mertlich D. Piloting home-based behavioural family systems therapy for adolescents with poorly controlled diabetes. Children's Health Care 2003;32:65-79. https://doi.org/10.1207/S15326888CHC3201_5

87. Clark M, Hampson SE. Implementing a psychological intervention to improve lifestyle self-management in type 2 diabetes. Patient Educ Counsel 2001;42:247-56. https://doi.org/10.1016/S0738-3991(00)00128-2

88. Simmons $D$, Hartnell $S$, Watts J, et al. Effectiveness of a multidisciplinary team approach to the prevention of readmission from acute glycaemic events. Diabet Med 2015;32:1361-7. http://dx.doi.org/10.1111/dme.12779

89. Hofstede G. Culture's Consequences. Comparing Values, Behaviours, Institutions and Organizations Across Nations. 2nd edn. Thousand Oaks, CA: Sage Publications, 2001

90. Cohen J. Statistical Power Analysis for the Behavioral Sciences. 2nd edn. Hillsdale, NJ: Lawrence Earlbaum Associates,1988. 


\section{Appendix A}

The following search terms were used to find studies:

(1) Adolescence OR adolescent OR youth OR young people OR young person, OR teen OR juvenile OR puberty

(2) Diabetes

(3) Program OR intervention OR randomised controlled trial OR RCT

(4) Peer OR family OR parent OR friend and

(5) Outcome OR efficacy OR control OR communicat OR social OR knowledge OR diet OR skill OR exercise. 


\section{Appendix B}

\section{Study characteristics Country of origin}

The majority of studies $(n=16)$ were conducted in the USA $(94.2 \%)$ and only one study ${ }^{63}$ was conducted in Sweden (5.8\%). These countries are classified as individualist and so can be considered broadly homogenous. ${ }^{89}$

\section{Study design}

Although all studies within this review were RCTs, only 14 reported the method of randomisation and the remaining three did not specify it clearly. ${ }^{56,59,60}$ Studies including RCTs in the review typically had one intervention group with a control group (58.8\%). A small number had one intervention group with two control/comparison groups (41.2\%).60,64-69

\section{Data collection}

Ten of the 17 studies $^{56-59,62,64,65,67,70,72}$ collected data at two time points (baseline and post-treatment); one study collected data at three time points (baseline, 3 and 6 months follow-up); 71 three studies collected data at four different time points (baseline, 3, 6 and 12 months; 66 baseline, 2, 6 and 12 months; ${ }^{63}$ and pre-treatment, 6 weeks, 3 and 6 months $\left.{ }^{60}\right)$; one study collected data at five time points (baseline, 6, 12, 18 and 24 months $^{61}$ ); and two studies collected data at seven time points (baseline, 3, 6, 9, 12, 15 and 18 months $\left.{ }^{68,69}\right)$.

\section{Baseline reported results}

Nine studies reported baseline differences between groups but no significant differences were noted. Of the remaining studies, one reported significant and non-significant differences at baseline, ${ }^{72}$ two reported significant differences between groups at baseline 65,66 and five did not specifically report baseline differences between groups. $57,59,63,70,71$

\section{Intervention characteristics Intervention length}

The duration of the peer/parent-based interventions ranged from 1 hour $^{70}$ to 3 weeks, ${ }^{62} 6$ weeks, $^{60,63} 8$ weeks, $^{59,71} 3$ months, ${ }^{64-66} 6$ months ${ }^{56-58,67-69,72}$ and 24 months. ${ }^{61}$

\section{Setting}

Reports were frequently explicit about where the interventions had been conducted. Hospital outpatient clinics ( $n=13,76.5 \%)$ were the most likely setting for interventions, followed by home and community-based settings (17.6\%) and a school based-setting (5.9\%). The most typical setting was the diabetes outpatient clinic

\section{Delivery of interventions}

Most interventions were delivered by a range of highly qualified professionals (eg, psychologist, therapist, health advisor or social worker). However, in some studies it was difficult to identify who delivered which part of the intervention.

\section{Skills training}

The majority of the interventions used some form of social, psychological, cognitive and behavioural skills training (82.4\%), followed by group therapy, independent problem solving (11.7\%) and lecture/discussion-based sessions (5.9\%).

\section{Intervention measures}

All studies (except the study by Newton and Ashley ${ }^{70}$ ) provided a full description of the intervention and used $\mathrm{HbA}_{1 \mathrm{c}}$ as the primary outcome measure, which was measured at baseline and at each follow-up. Validated measures were used to assess secondary outcomes, which varied between studies (see Table 1).

\section{Participant characteristics \\ Demographics}

Most studies reported the ethnicity of the participants with the exception of two studies. ${ }^{60,63}$ The sample size varied greatly from 11 to 127 (in each group). Details of sample size justification were only provided for five studies based on power analysis calculations. 58,61,63,70,72 Across the 17 studies, the total number of adolescents was 1,623. More than half of the studies involved fewer than 130 participants. Given that most of the studies involved an intervention group and a control group, subject numbers per condition tended to be sufficient. The mean age of adolescents across all the studies was 14.29 years and the mean duration of diabetes was 5.6 years. Only 13 studies reported attrition rates (4-37\%). 


\section{Appendix C}

\section{Effect sizes}

Effect sizes could be computed using Cohen's $d^{90}$ for $\mathrm{HbA}_{1 \mathrm{c}}$ in five of the 17 interventions and for psychosocial functioning and other outcome measures in 10. The remaining interventions had insufficient data (eg, no means or SDs) to calculate the effect sizes.

\section{HbA1c}

Negative effect sizes regarding $\mathrm{HbA}_{1 c}$ were indicated in Wysocki et a/68 at long-term follow-up periods. However, it is noted that $\mathrm{HbA}_{1 \mathrm{c}}$ for the Behavioural Family Systems Therapy (BFST-D) group was significantly lower than that of the standard care (SC) group at months 6, 9, 12, 15 and 18, and significantly lower than that of the Education and Support (ES) group at months 9, 15 and 18. Additionally, two more studies demonstrated negative effect sizes regarding $\mathrm{HbA}_{1 c} .{ }^{56,58}$ However, in the study by Ellis et al, ${ }^{56}$ adolescents receiving Multi-Systemic Therapy (MST) had significant improvements in metabolic control from study entry to 6 months post-test. Similarly, Ellis et al reported a significant change (ie, $\mathrm{HbA}_{1 \mathrm{c}}$ decreased $0.92 \%$ ) in youth in singleparent families assigned to MST. ${ }^{58}$

At 3-month follow-up, a multi-family (MF) group intervention plus parent simulation (S)-based study ${ }^{60}$ (three groups) was found to have a large effect size on metabolic control measured by the $\mathrm{HbA}_{1 \mathrm{c}}$. Further follow-up effect size was also quite large in this study when MF+S was compared with control at 6 months. The findings of the second and third cycles of this study also demonstrated a large effect size at 3 and 6 months.

Findings of a 3-month study ${ }^{65}$ demonstrated a small effect size on $\mathrm{HbA}_{1 \mathrm{c}}(0.28$ when BFST was compared with ES rather than current therapy $(\mathrm{CT}), 0.18)$. At 7 months the MST-based intervention ${ }^{57}$ was compared with SC using two approaches: intent to treat analysis and per-protocol analysis, where a medium effect size on $\mathrm{HbA}_{1 \mathrm{c}}(0.64)$ was found only in the per-protocol analysis.

\section{Psychosocial, diabetes management and adherence measures}

Negative effect sizes regarding psychosocial measures were indicated in the study by Wysocki et a/65 at the 3-month period. However, the BFST group improved significantly more on the overt conflict and deficit scale and on the extreme belief scale than the CT group. A significant main effect for groups on change in the DRC family composite scores was found, favouring the BFST group, but no significant main effects for the $\mathrm{SCI}$ and TADS. At 6 months the BFST-based intervention ${ }^{67}$ was found to have a moderate effect size on one of the subscales called family structure (0.48).

In a later study, Wysocki et a/68 at 6-month follow-up reported a medium effect size on the diabetes self-management profile (0.56). These results are indicated to be sustained at 1 year (0.6)

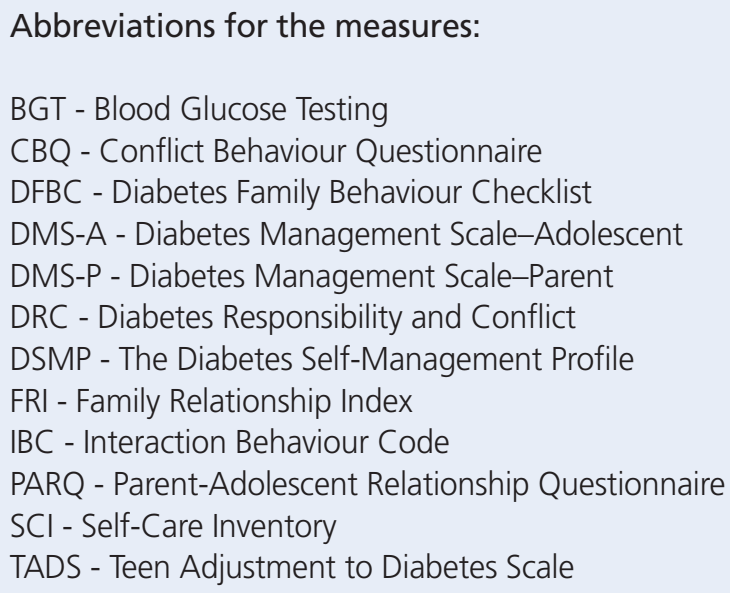

and at 18 months (0.36). This study had negative effect sizes related to the DRC scale at 6, 12 and 18 months. Furthermore, negative effect sizes were indicated in a further study by Wysocki et $a^{69}$ related to IBC for negative communication for all participants at 6, 12 and 18 months. However, it is noted that adolescents and mothers in the BFST-D group had significantly lower scores than the SC group (at all follow-ups) and ES at the 6-month follow-up only. For positive communication, high medium effect sizes were indicated in this study for:

1. Adolescents (at 6 months, 0.6; 12 months, 0.30; and 18 months, 0.54)

2. Mothers (at 6 months, 0.60; 12 months, 0.53; and 18 months, 0.61)

3. Fathers (at 6 months, $0.37 ; 12$ months, 0.66 ; and 18 months, $0.44)$.

A 5-8-week long home-based BFST53 indicated negative effect sizes on DRC and CBQ outcome measures for all participants. However, post treatment the BFST group (including all participants) scored much lower on the DRC and CBQ measures than the control group. At 7 months the MST-based intervention ${ }^{58}$ was found to have very small and large effect sizes on family functioning as measured by the FRI (0.02), DFBC (primary caregivers, 0.13 ) and DFBC (secondary caregivers, 0.94).

A web-based 7-week long intervention ${ }^{70}$ demonstrated small effect sizes for diabetes quality of life (0.19) and self-efficacy of diabetes management (0.21). However, Kichler and colleagues' 'Kicking in Diabetes Support' intervention'2 demonstrated small to medium effect sizes at 6 months post test on readiness to change the balance of responsibility scale (0.23), diabetes family relationship questionnaire $(0.28)$, paediatric quality of life inventory diabetes $(0.43)$ and diabetes family relationship questionnaire $(0.47)$. 
In terms of diabetes management and adherence-related factors, negative effect sizes were indicated in the study by Wysocki and colleagues ${ }^{65}$ on all IC, intensity, duration and diet amount scores at 3 months. This study also indicated small effect sizes on frequency (0.06), insulin (0.20) and exercise (0.17), a moderate effect size on diet composition (0.5) and a large effect size on testing/eating frequency (0.71). Moreover, another study56 indicated negative effect sizes at 6-month follow-up related to DMSA, DMS-P, EATADH (24-hour recall dietary adherence) and hospital admissions. However, there was a significant difference in hospital admission between the intervention and control groups at 6 months. This study also indicated a small effect size on BGTS (24-hour recall blood glucose testing adherence) (0.15) and moderate effect sizes on meter (0.36) and 24-hour recall insulin adherence (INSADH) (0.48). Furthermore, at 7 months an MST-based intervention ${ }^{57}$ was found to have moderate effect sizes on admissions (0.63 and 0.65) and large effect sizes on meter (1.09 and 1.01) and BGTS (0.83 and 1.05). A moderate effect size was indicated by Ellis et $a^{58}$ on BGT frequency (0.67) at 7 months post-test.

Overall, a variety of studies used diverse outcome measures. Some studies provided insufficient data to estimate accurate effect sizes. However, three outcome measures were consistently used across studies: (1) $\mathrm{HbA}_{1 \mathrm{c}}$ (6 studies); (2) PARQ (2 studies); and (3) DRC (3 studies). These studies had inconsistencies in the follow-up periods and had small sample sizes (see Table 1). It should be noted by looking at the individual effect sizes of the studies that two interventions ${ }^{68,69}$ were much longer in duration (eg, 18 months) than the remaining seven interventions. However, much larger effect sizes (at 3 months, 2.08; 6 months, 1.25) were found in the study by Satin et a/60 related to $\mathrm{HbA}_{1 \mathrm{c}} \mathrm{com}-$ pared with all the remaining studies. 